\title{
ABORDAGENS QUALITATIVAS: SUA CONTRIBUIÇÃO PARA A ENFERMAGEM
}

\author{
Dulce Maria Rosa Gualda* \\ Miriam Aparecida Barbosa Merighi** \\ Sonia Maria Junqueira Vasconcelos de Oliveira***
}

GUALDA, D.M.R et al. Abordagens qualitativas: sua contribuiçao para a enfermagem

Rev.Esc.Enf.USP., v.29, n.3, p.297-309, dez. 1995.

Este trabalho aborda algumas caracteristicas que configuram a melodologia qualitativa. Comenta sobre os métodos que tem sido mais utilizados, como: a fenomenologia, a teoria fundamentada nos dados, a elnografia e a pesquisa participante,ressalta a perspectiva de utilização destes métodos qualitativos para a enfermagem.

UNITERMOS; Metodologia qualitativa. Pesquisa em enfermagem.

\section{INTRODUÇÃO}

O ser humano tem como característica, a necessidade de entender e explicar o universo do qual faz parte. Este conhecimento se desenvolve através da relação que se estabelece entre um sujeito e um determinado objeto, esta relação apresenta-se de forma desodernada e oferece uma multiplicidade de impressões e facetas que podem ser captadas pelo sujeito sob diversas persperctivas. Para qualquer abordagem, entretanto, é necessário um ponto de referência. A existência de um referencial teórico metodológico estabelecido que delimite o campo de observação e determine as condições de relacionamento entre os fenomenos, é imperativo.

\footnotetext{
* Obstetriz. Professor Doutor do Departamento de Enfermagem Materno-Infantile Psiquiátrica da Escola de Enfermagem da ISP.

** Enfermeira. Professor Doutor do Departamento de Enfermagem Materno-Infantil e Psiquiátrica da Escola de Enfermagem da USP.

*** Enfermeira. Assistente do Departamento de Enfermagem Materno-Infantil e Psiquiátrica da Escola de Enfermagem da USP'.
} 
Nas últimas décadas, surgiu uma nova abordagem dos fenômenos, utilizada por humanistas, cientistas e filosofos, baseada numa perspectiva dinâmica e holística, na qual a pesquisa qualitativa surge como alternativa metodologica.

Metodologia qualitativa, no sentido lato, refere-se a pesquisa que produz dados descritivos: palavras tanto escritas como faladas, assim como comportamentos observáveis das pessoas. LEININGER ${ }^{\mathrm{s}}$ refere-se à pesquisa qualitativa como métodos e técnicas de observação, análise e interpretação de atributos, padrões, características e significado do fenômeno em estudo. De modo geral, a metodologia qualitativa enfatiza a importância de se conhecer, entender e interpretar acuradamente a natureza das situações e eventos quer sejam eles, passados ou presentes.

BOGDAN \& BIKLEN ${ }^{2}$, apresentam cinco características básicas que configuram a metodologia qualitativa:

1 - a pesquisa qualitativa tem o ambiente natural como fonte direta dos dados e o pesquisador representa o instrumento chave. Supõe um contacto direto e prolongado do pesquisador com o ambiente e a situação que está sendo investigada, através de um intenso trabalho de campo.

2. - os dados coletados são predominantemente descritivos. Assim sendo, o pesquisador precisa estar atento para o maior número possivel de elementos presentes na situação em estudo pois, um aspecto supostamente corriqueiro pode ser essencial na compreensão do fenômeno. Da mesma forma, os resultados são expressos em descrições e narrativas que tem como base a percepção de um fenômeno num contexto.

3. - a preocupação com o processo é maior do que com o produto. O interesse do investigador no estudo de um determinado fenômeno 6 verificar como ele se manifesta nas atividades, nos procedimentos e nas interações do dia a dia. A complexidade do cotidiano é sistematicamente retratada nas pesquisas qualitativa.

4 - o significado que as pessoas dão as coisas e a sua vida, são focos de atenção especial do pesquisador. $\Lambda$ tentativa é de captar a "perspectiva dos participantes" isto é, a maneira que encaram as questões que estão sendo focalizadas.

5 - a análise dos dados tende a seguir um processo indutivo. A não existência de hipoteses ou questões especificas formuladas apriori não implica na não existência de um quadro teórico que oriente a coleta e análise de dados. Os significados, a interpretação e a análise surgem da percepção do fenômeno visto num contexto.

LEININGER ${ }^{9}$ complementa as afirmações anteriores enfatizando que a metodologia qualitativa na enfermagem tem como foco principal a natureza, a essência, o significado e os atributos do fenômeno que está sendo estudado; seu âmbito é geralmente amplo, holístico e compreensível. No processo de 
investigações poucos dados são excluídos, pois a meta é conhecer a totalidade dos aspectos gerados nas circunstâncias culturais, ambientais e sociais que revelam interações humanas, símbolos, valores, estilos de vida e visão de mundo. O que se busca, na verdade, são dados objetivos e subjetivos para a obtenção da verdade das pessoas.

TRIVIÑOS ${ }^{18}$ tece também algumas consideraçōes importantes sobre a metodologia em questão. Afirma que não há uma sequência rígida das etapas assinaladas, cita como exemplo, a coleta e análise de dados salientando que as informações obtidas, quando interpretadas, podem gerar novas buscas de dados. Isto se deve ao fato do pesquisador não iniciar o seu trabalho orientado por hipoteses levantadas "a priori", onde todas as alternativas são verificadas empiricamente. Comenta ainda que as hipoteses estabelecidas podem, a qualquer momento, ser deixadas de lado criando-se outras, a partir de informações obtidas numa primeira etapa. Não obstante, o pesquisador inicie sua investigação apoiado numa fundamentação tébrica geral, o que demanda uma revisão aprofundada da literatura sobre o tópico em estudo. A maior parte do trabalho, neste aspecto, se realiza no processo de investigação. Assim sendo, a necessidade da teoria surge face às interrogações que se apresentam. $\mathrm{Na}$ pesquisa qualitativa existe pouco empenho na definição operacional das variáveis, que são apenas discretas mas podem ser em grande numero. Outro aspecto que o autor salienta é a despreocupação do pesquisador com o tratamento estatístico dos dados e a determinação do tamanho da amostra. Salienta ainda que o pesquisador orientado pelo enfoque qualitativo tem ampla liberdade teórico metodológico para realizar seu estudo e os limites de sua iniciativa particular estão exclusivamente fixados pela exigência de um trabalho cientiffico.

Assim, c cada vez mais evidente o interesse que os enfermeiros pesquisadores vem demonstrando pelo uso das metodologias qualitativas. Dentre os métodos que tem sido mais utilizados destacamos: a fenomenologia, a teoria fundamentada nos dados, a etnografia e a pesquisa participante.

\section{PESQUISA FENOMENOLÓGICA}

A fenomenologia representa uma tendência dentro do idealismo filosófico e dentro deste, ao denominado idealismo subjetivo.

Segundo MARTINS", ela representa o estudo dos significados, das essências articuladas no discurso do sujeito, atravês do qual o fenômeno vai se mostrar.

O movimento fenomenologico nasceu da erença de que o comportamento das pessoas não pode ser controlado, aceita a experiência na forma que existe na consciência do individuo. $\Lambda$ intuição se torna importante ao desenvolvimento do conhecimento através do senso de impressão. 
O primeiro requisito da fenomenologia é que não haja noções préconcebidas, expectativas ou modelos de referência presentes para guiar os pesquisadores. Na medida que eles começam a analisar os dados, tem-se uma experiência de vida, uma visão de mundo, e isso é o que gera a interrogação. Portanto, o recurso utilizado é a interrogação através do sujeito e a trajetória é uma decorrência de todo "mundo vida" do pesquisador.

Em pesquisa fenomenologica, o pesquisador não tem um problema para pesquisar, tem dúvidas sobre as quais interroga; ao interrogar constroi uma trajetória, pela qual caminha em direção ao fenômeno, naquilo que se manifesta por si através do sujeito que experiencia a situação.

$O$ início do enfoque fenomenologico deve fundamentar-se no conceito de fenômeno, que está oculto e não se mostra a menos quando interrogado, utiliza como instrumento de conhecimento a intuição, a reflexão e a descrição.

Nesta abordagem, a forma de compreender a experiência não se assemelha aquela pela qual se conhece a realidade física ou biológica. Por esta razão, a fenomenologia centraliza-se no significado do fenômeno, através da análise do significado e relevância de uma experiência da pessoa e através de um conhecimento da essência dos fenômenos e da consciência que 6 indispensável para a compreensão.

O foco central da fenomenologia é a descrição do fenômeno e não sua explicação, não se preocupando com as relaçōes causais. A ênfase está no sentido de mostrar c não demonstrar e a descrição rigorosa do fenômeno prevê o alcance de sua essência.

Assim, a fenomenologia busca:

- descrever experiências vividas da maneira mais fiel que possível (descarta do pensamento as questões concretas da existência).

- questionar constantemente as consequências de quaisquer pressupostos e hipóteses.

- compreender as formas e os meios que permitem ao ser humano adquirir experiência, interpretar o mundo e atuar dentro dele.

- descrever acuradamente a experiência do 'enômeno em estudo e não gerar teorias ou modelos para desenvolver explicação geral.

Para o pesquisador a vantagem do método fenomenologico é obter uma compreensão maior do fenômeno estudado. Para os sujeitos a vantagem é a mudança que neles se opera; a reflexão mais profunda, a melhor compreènsão da sua própria existência as modificações com vistas ao crescimento e as possibilidades de mudanças.

Pode-se destacar como implicaçoes importantes da pesquisa fenomenologica para a enfermagem:

- O método fenomenologio é adequado para a enfermagem pois as respostas são dadas por pessoas que vivem o fenômeno, que o experiencia. 
- A enfermagem implica cm um encontro especial de pessoas. É uma experiência intersubjetiva em que ocorre um verdadeiro partilhar.

- Uma das responsabilidades do enfermeiro em relação a sociedade é a orientação dos indivíduos e da família, a escolha de possibilidades, no processo mutável de saúde. Isso é conseguido através da participação intersubjetiva com as pessoas e suas familias.

- A prática da enfermagem envolve inovações e criatividade e não regras prescritivas, as metas da enfermagem facilitam a qualidade de vida a partir da perspectiva da pessoa.

- A fenomenologia não vai explicar a doença, ela busca compreender o homem em sua totalidade existencial, encuanto homem inserido numa dada sociedade, histórica e culturalmente situada.

- funções, desempenhos, papeis, sistema de organização, quando estudados pela enfermagem. devem recolocar tais questóes na visão centrada no sujeito enquanto pessoa portadora de uma dimensão e valor ontologico proprios, sujeito concreto responsável pela sua vida e não mais numa abordagem que o coloque como objeto - coisa, número ou dimensão de anonimato.

Portanto, é inegável a contribuição da fenomenologia para a enfermagem uma vez que a tendência desta 6 a de retomar e considerar o homem em seu todo, de modo holístico, e não mais isoladamente em partes. A assistência à saúde volta-se assim, para pessoas congruentes e livres e não mais para pacientes anônimos sob os quais se irá atuar.

\section{TEORIA FUNDAMENTADA NOS DADOS}

A teoria fundamentada nos dados foi desenvolvida por dois sociologos Bsarney (ilaser e Ansclm Strauss. nos anos sessenta. Representou uma reação de contraposição à corrente sociologica da época que enfatizava a construção de teorias de alto nível e abstração conceitual, desvinculadas da pesquisa, com ênfase nos aspectos técnicos dos métodos quantitativos. Com o objetivo de preencher a lacuna entre teoria e pesquisa, a teoria fundamentada nos dados enfatiza o processo de geração de teoria a partir de dados coletados e analisados sistematicamente. Assim, a teoria fica ligada ou fundamentada, aos dados através de exemplos descritivos e as evidências empíricas. Desta forma a teoria fundamentada nos dados serve de estrutura conceitual que viabiliza a construção de hipoteses testáveis.

As técnicas para coleta e análise de dados nesta metodologia são semelhantes as da pesquisa de campo e da etnografia, que consiste de entrevista e observação participante. Preconiza a manutenção de registros sistemáticos e detalhados clas observações e análise simultânea dos dados coletados. Difere porém, da pescluisa etnográfica, pois esta, enfatiza a análise 
na perspectiva cultural e utiliza abordagens teóricas diversificadas. $A$ teoria fundamentada nos dados enfoca no desenvolvimento de papéis, e no relacionamento sob o ponto de vista do interacionismo simbolico. COULON ${ }^{4}$ afirma que na perspectiva do interacionismo simbólico, a concepção que os autores fazem para si do mundo social constitui em última análise o objeto essencial da pesquisa sociologica.

Segundo STERN ${ }^{16}$ a teoria fundamentada nos dados difere de outras metodologias de pesquisa em vários aspectos:

- a estrutura conceitual o gerada nos dados ao invés de serem utilizados estudos anteriores, muito embora, de alguma forma influenciem a pesquisa;

- o pesquisador busca descobrir os processos dominantes no cenário cultural. ao invés de descrever as unidades de estudo:

- as partes dos dados são comparadas entre si ao invés de serem comparadas aos índices totais. Por esta razão, foi denominada "análise qualitativa comparativa";

- a coleta dos dados pode ser modificada de acordo com a evolução da teoria;

- o investigador ao invés de seguir uma série de passos lineares, trabalha com uma matriz na qual os vários processos de pesquisa estão operando de uma só vez. Assim sendo, o investigador examina os dados na medida que são coletados e começa a codificar, categorizar, conceituar e escrever as primeiras reflexões, que são incluídas posteriormente no relatório de pesquisa.

De acordo com (aIASSER; STRAUSS7, a teoria é gerada por um processo de indução, onde as categorias analíticas emergem dos dados e são elaboradas no decorrer da pesquisa.

TAYLOR; BOGDAN17 comentam que este metodo não tem o objetivo de procurar evidências que comprovem hipoteses pre-estabelecidas, mas sim, formar abstrações a partir da análise dos dados.

O passo final da pesquisa consiste na produção e apresentação de uma teoria substantiva, fundamentada $e$ apoiada nos dados da investigação onde a literatura 6 utilizada apenas para explicar a teoria.

A teoria fundamentada nos dados representa uma abordagem qualitativa que permite estudar o universo empírico da prática de enfermagem que inclui o estudo do processo-saúde-doença, nas suas diversas perspectivas.

\section{PESQUISA ETNOGRÁFICA}

A etnografia é uma metodologia qualitativa de pesquisa que teve sua origem na antropologia cultural. Contudo, outras áreas do conhecimento a tem utilizado, com o objetivo de compreender a natureza humana, inclusive a enfermagem. 
A etnografia é tanto processo como produto da investigação e tem como objetivo descrever uma cultura ou aspectos de uma determinada cultura. Como processo, envolve uma atitude de aprender com, ao invés de estudar as pessoas. A finalidade é captar o ponto de vista nativo de indivíduos de um grupo cultural. Assim sendo, o processo metodológico fundamenta-se no conceito de cultura, sendo conduzido at ravés de um intenso trabalho de campo.

O principal método de coleta de dados é a observação participante, embora, outras técnicas como entrevista, conversas informais,análise documental e história de vida, sejam conjuntamente utilizados.

Segundo BECKER' o observador participante coleta dados através de sua participação na vida cotidiana do grupo ou organização que estuda. Ele observa as pessoas que está estudando para ver as situações com que se deparam normalmente c como se comportam diante delas. Entabula conversação com alguns ou com todos os participantes desta situação e descobre as interpretações que eles tem sobre os acontecimentos que observou.

No método etnográfico o pesquisador é considerado o principal instrumento e tem a flexibilidade de decidir quando, onde e como coletar os dados. As técnicas preconizadas servem para urientar o pesquisador e não são normas rígidas nem preceiturários a serem cumpridos ininterruptamente. O trabalho de campo demanda a presença constante do pesquisador no ambiente cultural, vivendo, participando do cotidiano, anotando sistematicamente e validando as observações e os dados das entrevistas.

Diferentemente de outros metodos de pesquisa, nos quais os dados são primeiramente coletados e posteriormente analisados, nas pesquisas etnográficas os dados são coletados e analisados simultaneamente, visando obter respostas as questões que surgem na busca da compreensão do modo de vida das pessoas, na sua propria perspectiva. O processo de questionar e responder sobre o que é relevante ao contexto, conduz a investigação através de ciclos até que atinja os objetivos propostos, ou seja, construir a teoria geral da cultura estudada. Não obstante, segundo SPRADLEY 14 todas as etnografias são consideradas obras inacabadas, quer tenham elas inúmeros volumes ou seja apenas um artigo.

$\Lambda$ finalidade da análise dos dados ex extrair temas e obter um entendimento profundo dos valores e crenças que guiam as ações dos individuos. Analisar dados à luz da etnografia envolve, abstração, generalização e in terpretação. Padrões culturais e evidências empíricas devem ser descritas e o significado para as pessoas, nas diversas situações sociais deve ser interpretado. O trabalho etnográfico que descreve os padrões culturais sem interpretar o significado é considerado incompleto.

GERMAIN ${ }^{\epsilon}$ tem se dedicado a essa modalidade de pesquisa e afirma que o local aprop riado para a realizaçio da etnografia 6 onde existem pessoas e atividades de enfermagem e de cuidado a saude, que requerem abordagem 
num contexto holístico. O autor complementa dizendo que é inegável a contribuição da etnografia para a enfermagem, por esta razão ela deve ser amplamente utilizada. O enfermeiro enquanto pesquisador etnográfico tem algumas facilidades no desenvolvimento do seu trabalho que podem ser aplicáveis, a pesquisa qualitativa de um modo geral destacando-se:

- o conhecimento das instituições de saúde;

- o treinamento nas atividades de observação e entrevista;

- o contato direto com diferentes subculturas;

- a experiência de lidar com questões relacionadas ao cuidado da saúde e da doença.

\section{PESQUISA PARTICIPANTE}

Sabe-se que nas pesquisas tradicionais, apesar do problema ser estudado e analisado criteriosamente, seu enfogue é unilateral, visualizado pelo ponto de vista do pesquisador. Este é considerado como alguém de con dição superior que analisa e questão de fora, sem vivenciála. Desta forma, não consegue atingir e envolver os pesquisados para aplicar achados que foram evidenciados.

Na enfermagem, como nas outras áreas, são realizadas btimas pesquisas, com importantíssimos resultados, mas que raramente chegam ao conhecimento da população pesquisada. Estes trabalhos tem sido desenvolvidos através do método tradicional positivista de pesquisa, que se limita a classificar, relacionar, descr vver e constatar situações ou eventos; buscando apenas o conhecimen to científico sem a pretenção de intervir sobre o problema estudado. Segundo MADEIRA10 há um distanciamento entre o pesquisador e os pesquisados para que não haja "interferência" na pesquisa.

Entretanto, um novo tipo de pesquisa proveniente da sociologia $e$ antropologia, com aplicação em várias áreas vem sendo desenvolvida, a metodologia humanista, que tem sua base no materialismo dialético. Conforme BRUSCHINI \& BARROSO3, nesta metodo'ogia, o processo e o produto da pesquisa são tomados de forma integrada, gerando resultados que são utilizados na transformação da realidì de estudada. O seu objetivo é trabalhar em estreita interação com os sujeitos pesquisados, numa relação mútua de aprendizado em todas as etapas do processo.

Nesta metodologia, ainda, os pescluisadores são considerados sujeitos da investigação, o pesquisador não é o dono do saber e da verdade e para que seus resultados sejam efetivos requer o envolvimento de pesquisador pesquisado. A preocupação do método é a qualidade do fenômeno e não a quantidade de sua ocorrência e, através da ação-reflexão-ação procura constantemente, atuar e modificar a situação estudada. 
$\mathrm{DEMO}^{5}$ ao discorrer sobre algumas definições de pesquisa participante cita algumas caraterísticas apontadas por Tandon.

a) É um processo de conhecer e agir. $\Lambda$ população engajada na pesquisa participante simultancamente aumenta seu entendimento e conhecimento de uma situação particular, bem como parte para uma ação de mudança em seu beneficio.

b) É iniciada na realidade concreta que os marginalizados pretendem mudar. Gira em torno de um problema existente. Caso haja consciência suficiente, a própria população inicia o processo e pode até mesmo dispensar o perito externo. Mas, ainda começando pelo perito, o envolvimento da população 6 essencial.

c) Variam a cxtensão e a natureza da participação. No caso ideal a população participa do processo inteiro: proposta de pesquisa, coleta de dados, análise $\mathrm{e}$ in tervenção na realidade.

d) A população deve ter o controle do processo.

c) Tenta-se eliminar ou pelo menos reduzir as limitações da pesquisa tradicional. Pode-se empregar métodos tradicionais na coleta de dados, mas enfatiza posturas qualitativas e hermenêuticas e a comunicação interpessoal.

f) E um processo coletivo.

g) Euma ação educativa."

Para MENDONÇA ${ }^{12}$ a ação educativa é capaz de desenvolver nas pessoas a consciência crítica das causas dos problemas e ao mesmo tempo, de criar uma prontidão para atuar no sentido da mudança.

Conhecemos a gama de problemas e as dificuldades que os profissionais de enfermagem enfrentam no dia a dia. No contexto hospitalar, principalmente, observamos uma grande discrepancia, entre o que se aprende, o que se acredita estar correto e aquilo que realmente se faz. Estas incoerências, freqüentemente, levam o profissional a ter um alto nível de insatisfação no trabalho mas, ao mesmo tempo, acomoda-se às situações. $O$ processo de pesquisa participante favorece o crescimento dos profissionais, através da reflexão conjun ta sobre o trabalho que desenvolvem $e$ suas relações com a melhoria das condiçōes de saúde da população.

Pode-se concluir, portanto, com base nas afirmações anteriores que qualitativo refere-se a experiência humana, sentida, vivida, processada, no scu contexto proprio, nas diversas situações sociais.

$\Lambda$ pesquisa qualitativa, como decorrência, tem a finalidade de compreender as experiências no seu todo, na perspectiva dos participantes. Para tanto, desenvolve-se em um processo essencialmente interativo, no qual as pessoas estudadas falam por si mesmas e ensinam ao pesquisador sobre suas vidas. Essa metodologia além do envolvimento demanda do pesquisador uma postura aberta e flexível para a descoberta do inesperado. 
Cabe salientar que a pesquisa qualitativa não é apenas um método de pesquisa, são métodos que guardam $\mathrm{cm}$ si características comuns e especificidades relativas à tradição histórica, orientação teórica e metodológica

Os métodos qualitativos são essencialmente diferentes dos métodos quantitativos. No entanto, tem havido uma tendência dos pesquisadores de avaliar pesquisas qualitativas com critérios da pesquisa quantitativa, SANDELOWSKY ${ }^{13}$ afirma que a questão do rigor metodológico da pesquisa qualitativa ainda não atingiu um consenso entre os pesquisadores.

GUBA \& LINCOLN ${ }^{8}$, discutem quatro critérios associados aos testes de rigor da pesquisa quantitativa que podem ser adaptados a pesquisa qualitativa: credibilidade, adequação, verificabilidade e confirmação.

Diz-se que um estudo tem credibilidade quando são apresentadas descrições ou interpretações fieis da experiência humana, de tal modo que as pessoas envolvidas poderiam reconhecề-las como proprias, de imediato.

$O$ critério de adequação é preenchido quando os achados do estudo se ajustam à contextos fora da situação esitudada ou quando são vistos por outras pessoas como significativos e aplicaveis em termos de suas próprias experiências. Considera-se ainda, que os resultados do estudo são condizentes com os dados do qual são derivados.

Na pesquisa qualitativa é enfatizada a questão da singularidade da experiência humana. Neste sentido, uma situação ou acontecimento não pode ser recriada. Para tanto, a verificabilidade 6 atingida quando outro pesquisador ou pesquisadores podem claramente seguir a "pista de decisão" utilizada pelo investigador do estudo. Além disso. outro pesquisador poderia chegar aos mesmos resultados ou resultados comparáveis, nunca contraditórios, com os mesmos dados, perspectivas e situações apresentadas.

Para SANDELOWSKY ${ }^{13}$ a verificabilidade é alcançada pela descrição, explanação ou justificação dos seguintes pontos:

- como o pesquisador tornou-se interessado pelo problema;

- como o pesquisador enxerga o fenômeno;

- propósito específico;

- como o assunto ou parte da cvidencia se incluiram e como foram abordados;

- o impacto que o sujeito e o pesquisador tiveram um para com o outro;

- como os dados foram coletados;

- quanto tempo demorou a coleta de dados;

- natureza dos locais no qual os dados foram coletados;

- como os dados foram reduzidos ou transformados para análise, interpretação ou apresentação;

- como vários elementos dos dados foram ponderados; 
- a exclusividade das categorias desenvolvidas para conter os dados;

- as técnicas especificas usadas para determinar o verdadeiro valor e a aplicabilidade dos dados.

()s estudos qualitativos valorizam a subjetividade. Neste aspecto, tornasc relevante o envolvimento do pesquisador com os sujeitos, sua realidade subjetiva e o significado que dão às suas experiências de vida. Assim sendo, o envolvimento é considerado um requisito essencial. Para tanto, a confirmação refere-se aos dados em si e não à subjetividade ou objetividade do pesquisador. ficando assim relacionada aos critérios anteriores: credibilidade, adequação e verificabilidade.

\section{CONTRIBUIÇÕES PARA A ENFERMAGEM}

() uso da metodologia qualitativa na enfermagem demonstra ter potencial de explorar novas áreas do conhecimento e do abordar perspectivas sobre a natureza da enfermagem.

$\Lambda$ descoberta do fenômeno de enfermagem no seu proprio contexto e a oportunidade de diretamente clarificar, observar e validar os dados, através do uso desta metodologia pode representar uma fonte de dados muito rica.

Em estudo sobre a pescuisa qualitativa em enfermagem no Brasil, STEFANELLLI et a $\left.\right|^{15}$ constataram que a mesma tem aceitação dos profissionais da área e, ainda, que os enfermeiros estāo realizando estudos desta natureza.

Uma das grandes preocupações dos enfermeiros diz respeito a definição da profissão, o objeto e finalidade de seus agentes, representando estes clementos importantes na realidade profissional. P'artindo-se do pressuposto que a prática norteia a pesquisa, a metodologia qualitativa ajuda a revelar a natureza e os atributos da enfermagem por possibilitar a compreensão do fenômeno.

Ém relação às perspectivas da pesquisa qualitativa para a enfermagem, acreditamos que:

- A pesquisa qualitativa contribui para a compreensão holística do homem.

- A pesquisa qualitativa oferece oportunidade de discussão de temas que devem ser exploradas de modo profundo.

- () estudo qualitativo o uma das alternativas que permite explorar melhor alguns problemas sobre a assistência de enfermagem.

- Abre novos horizontes para a enfermagem levando o enfermeiro (pesquisador) a refletir mais.

- A pesquisa qualitativa contribui para a compreensão do "ser" enfermeiro, $e$ para o desenvolvimento da enfermagem como ciência e profissão. 
A enfermagem exige de seus profissionais, além da competência técnica, a capacidade criativa, de reflexão, de análise crítica e um aprofundamento constante de seus conhecimentos, de modo a permitir o seu engajamento na realidade social. Há várias alternativas metodológicas e muitas lacunas para serem preenchidas. A pesquisa qualitativa, por sua característica própria, aponta muitas perspectivas no sentido de veicular novas idéias e novos planos, para construção sistemática de um corpo de conhecimentos de enfermagem e promover impacto na sua prática profissional.

Esperamos que a pesquisa qualitativa produza resultados cada vez mais suficientes para pensarmos nas soluções mais adequadas aos nossos problemas. Problemas esses que, pela sua natureza especifica, requerem técnicas de estudo especialmente adequadas.

GUALDA, D.M.R. et al. Qualitative approaches: contribuition for nursing.

Rev.Esc. Enf.USP., v.29, n.3, p.297-309, dec. 1995.

This paper focus on some characteristics of the qualitative methodology. Some of these methods are explored such as: participatory research, phenomenology,grounded theory and ethnography critical theory Perspectives of their utilization in nursing research are examined.

UNITERMOS: Qualitative methodology.Nursing research

\section{REFERÊNCIAS BIBLIOGRÁFICAS}

BECKER, H.S. Métodos de pesquisa em ciéncias sociais. 2ed. Sao Paulo, HUCITEC, 1994: cap. 2, p.47-64: Problemas de inferência e prova na observação participante.

BOGDAN, R.; BIKLEN, S.K. Qualitative research for education:an introduction to theory and methods. Boston, Allyn and Bacon, 1992.

BRUSCHINI, C.; BARROSO, C. Caminhandojuntas: uma experiencia em educaça sexual na periferia de São Paulo Cad.pesq., N.45, P. 43-9, 1983.

COULON, A. Etnometodologia . Petrópolis, Vozes, 1995, (ap. 1, p.9.17: Os precursores.

DEMO,P. Pesquisa participante: mito e realidade. Rio de Janeiro, SENAC, 1984. Cap, 3, p. 73-4: elementos metodológicos da P.P. 
GERMAN, C. Ethnography: the method In MUNHALL, P.L.; OILER. C.J. Nursing research: a qualitative perspective. Norwalk, Appleton Century Crofs cap.8, p. 147-62.

GLAZER, B.G.; ST'RAUSS, A.L. The discovery of grounded theory. New York, Aldine, 1967.

GUBA, E.G; LINC()LN, Y.S. Effective Evaluation. Sno Francisco, dossey-Bass, 1981.

LEININGER,M.M. Qualitative research methods in nursing. London, Grune \& Stratton, 1984

MADEIRA, L.M. Pesquisa participante: uma alternativa na enfermagem. Rev.Bras.Enf., Brasilia,v. 37, n.3/4. p.188-94, 1984 .

MARTINS, J. Psicologia fenomenológica. In: MARQUES, J. P'sicologia educacional p. 124-52.

MENI)ONÇA,G.F. Ação educativa nos serviços básicos de saúde. In: ENCONTRO DE EXPERIENCIAS DE EIJCAÇÃO E SAUUDE DA REGIÃO NORDESTE,Natal, 1982. Anais. Brasília, Centro deLocumentação do Ministério da Saúde, 1982. p.9-14.

SANDEL()WSKY, M. The problem of rigor in qualitative research.ANS-Adv.Nurs.Sci., v.8, n.3, p. $27-37,1986$.

SPRADLEY,d. The ethonographic interview. New York, Holt Renehart and Winston, 1979:

STEFANELLI, M.( . et al. Pesquisa qualitativa em enfermagem no Brasil. In:ENCONTRO INTERAMERICANO DE PESQUISA QUALITATIVA EM ENFERMAGEM, 1., SaoPaulo, 1988. Anais. Săo Paulo, Escola de Fnfermagem da USP, 1988. p.13.

STERN, P'. Grounded theory methodology, Its uses and processes. Image. v.12, n.1, p.20-3, 1980.

TAYLOR,S.J.;BOGDAN, R. Introduction to qualitative research methods: the search for meanings. Now York, John Wiley \& Sons, 1984. cap 1, p.1-12: Go to the people.

TRIVIN()S, A.N.S. Introducano a pesquisa em ciências sociais. Sno Paulo, Atlas, 1987. 